\title{
Brain Injury Lesion Imaging Using Preconditioned Quantitative Susceptibility Mapping without Skull Stripping
}

\author{
(D) S. Soman, (D). Liu, (D) G. Kim, (D). Nemec, DS.J. Holdsworth, D K. Main, DB. Lee, DS. Kolakowsky-Hayner, (D) M. Selim, \\ (D) A.J. Furst, (DP. Massaband, (D). Yesavage, (D) M.M. Adamson, DP. Spincemallie, (DM. Moseley, and (D). Wang
}

\begin{abstract}
BACKGROUND AND PURPOSE: Identifying cerebral microhemorrhage burden can aid in the diagnosis and management of traumatic brain injury, stroke, hypertension, and cerebral amyloid angiopathy. MR imaging susceptibility-based methods are more sensitive than CT for detecting cerebral microhemorrhage, but methods other than quantitative susceptibility mapping provide results that vary with field strength and TE, require additional phase maps to distinguish blood from calcification, and depict cerebral microhemorrhages as bloom artifacts. Quantitative susceptibility mapping provides universal quantification of tissue magnetic property without these constraints but traditionally requires a mask generated by skull-stripping, which can pose challenges at tissue interphases. We evaluated the preconditioned quantitative susceptibility mapping MR imaging method, which does not require skull-stripping, for improved depiction of brain parenchyma and pathology.
\end{abstract}

MATERIALS AND METHODS: Fifty-six subjects underwent brain MR imaging with a 3D multiecho gradient recalled echo acquisition. Mask-based quantitative susceptibility mapping images were created using a commonly used mask-based quantitative susceptibility mapping method, and preconditioned quantitative susceptibility images were made using precondition-based total field inversion. All images were reviewed by a neuroradiologist and a radiology resident.

RESULTS: Ten subjects (18\%), all with traumatic brain injury, demonstrated blood products on 3D gradient recalled echo imaging. All lesions were visible on preconditioned quantitative susceptibility mapping, while 6 were not visible on mask-based quantitative susceptibility mapping. Thirty-one subjects (55\%) demonstrated brain parenchyma and/or lesions that were visible on preconditioned quantitative susceptibility mapping but not on mask-based quantitative susceptibility mapping. Six subjects (11\%) demonstrated pons artifacts on preconditioned quantitative susceptibility mapping and mask-based quantitative susceptibility mapping; they were worse on preconditioned quantitative susceptibility mapping.

CONCLUSIONS: Preconditioned quantitative susceptibility mapping MR imaging can bring the benefits of quantitative susceptibility mapping imaging to clinical practice without the limitations of mask-based quantitative susceptibility mapping, especially for evaluating cerebral microhemorrhage-associated pathologies, such as traumatic brain injury.

ABBREVIATIONS: $\mathrm{CMH}=$ cerebral microhemorrhage; GRE = gradient recalled-echo; $\mathrm{mQSM}=$ mask-based $\mathrm{QSM} ; \mathrm{pQSM}=$ preconditioned $\mathrm{QSM} ; \mathrm{QSM}=$ quantitative susceptibility mapping; $\mathrm{TBI}=$ traumatic brain injury

M ultiple diseases, including traumatic brain injury $(\mathrm{TBI}),{ }^{1,2}$ stroke, ${ }^{3,4}$ hypertension, and cerebral amyloid angiopathy ${ }^{5}$ require assessment of cerebral microhemorrhage $(\mathrm{CMH})$ for $\mathrm{di}-$ agnosis and/or management. However, the choice of neuroimag-

Received August 18, 2017; accepted after revision December 4.

From the Departments of Radiology (S.S., G.K., B.L.) and Neurology (M.S.), Beth Israel Deaconess Medical Center, Harvard Medical School, Boston, Massachusetts; Department of Biomedical Engineering (Z.L., Y.W.), Cornell University, New York, New York; Department of Biomedical Imaging and Image-Guided Therapy (U.N.), Medical University of Vienna, Vienna, Austria; Departments of Radiology (S.J.H., P.M., M.M.), Psychiatry and Behavioral Sciences (A.J.F., J.Y., M.M.A.), and Neurosurgery (M.M.A.), Stanford University, Stanford, California; Research Division, Defense and Veterans Brain Injury Center (K.M.), General Dynamics Health Solutions, Silver Spring, Maryland; Department of Rehabilitation Medicine (S.K.-H.), Icahn School of Medicine at Mount Sinai, New York, New York; Departments of Psychiatry (A.J.F., J.Y.) and Radiology (P.M.) and Defense and Veterans Brain Injury Center (M.M.A.),

ing technique can provide very different assessments of $\mathrm{CMH}$ burden. While knowledge of $\mathrm{CMH}$ burden and distribution should inform risk stratification and treatment choices, variabil-

VA Palo Alto Health Care System, Palo Alto, California; and Department of Radiology (P.S., Y.W.), Weil Cornell Medical College, New York, New York.

Paper previously presented, in part, at Annual Meeting of the American Society of Neuroradiology and the Foundation of the ASNR Symposium, April 22-27, 2017; Long Beach, California; and International Society for Magnetic Resonance in Medicine Annual Meeting and Exhibition, April 22-27, 2017; Honolulu, Hawaii.

This study was funded by the War Related Illness and Injury Study Center, National Institutes of Health RO1 EB013443, RO1 NS090464, R01 NS095562, R01 CA181566, and R01CA178007.

Please address correspondence to Salil Soman, MD, Rosenberg B90A, 1 Deaconess Rd, Boston, MA 02215; e-mail: sal@alum.mit.edu

- Indicates open access to non-subscribers at www.ajnr.org

http://dx.doi.org/10.3174/ajnr.A5550 
Table 1: Subject demographics across Ohio TBI Scores ${ }^{23}(n=56)$

\begin{tabular}{|c|c|c|c|c|c|c|c|c|}
\hline \multirow[b]{2}{*}{ Ohio TBI Score } & \multicolumn{2}{|c|}{ Sex } & \multicolumn{3}{|c|}{ Age (yr) } & \multirow{2}{*}{$\begin{array}{l}\text { Blood on } \\
\text { GRE }\end{array}$} & \multicolumn{2}{|c|}{ Nonvisualized Brain } \\
\hline & Male & Female & Mean Age & SD & Age Range & & on mQSM & on pQSM \\
\hline All & 34 & 22 & 42.1 & 14.42778 & $20-74$ & 10 & 31 & 5 \\
\hline 1 (none) & 13 & 9 & 41.7 & 14.6878 & $20-74$ & 1 & 13 & 3 \\
\hline 2 (mild) & 2 & 2 & 44.0 & 15.71623 & $27-58$ & 1 & 3 & 0 \\
\hline 3 (mild) & 8 & 5 & 47.0 & 14.94434 & $30-71$ & 4 & 8 & 1 \\
\hline 4 (moderate) & 2 & 1 & 45.7 & 13.50309 & $32-59$ & 0 & 1 & 0 \\
\hline 5 (severe) & 9 & 5 & 37.9 & 13.96621 & $23-64$ & 4 & 5 & 1 \\
\hline $\begin{array}{l}\text { Correlation across TBI } \\
\text { severity }\end{array}$ & \multicolumn{2}{|c|}{$\chi^{2}=0.17, P=.98^{\mathrm{a}}$} & \multicolumn{3}{|c|}{ ANOVA $F(3,52)=0.74, P=.53$} & $P=.11^{\mathrm{b}}$ & $\chi^{2}=2.20, P=.53^{\mathrm{a}}$ & $P=.88^{\mathrm{b}}$ \\
\hline $\begin{array}{l}\text { Correlation with blood } \\
\text { products on 3D GRE }\end{array}$ & \multicolumn{2}{|c|}{$\chi^{2}=0.04, P=.86^{\mathrm{a}}$} & \multicolumn{3}{|c|}{$P=.49^{c}$} & - & $\chi^{2}=2.99, P=.08^{\mathrm{a}}$ & $P=.10^{b}$ \\
\hline
\end{tabular}

ity in $\mathrm{CMH}$ assessment based on the choice of neuroimaging method produces uncertainty about how this information should be used to inform patient management. ${ }^{6}$ This ambiguity signals the need for an easy-to-implement, sensitive, and specific method to identify CMHs.

Susceptibility-based $\left(\mathrm{T}^{*}\right.$ ) MR imaging methods (2D gradient recalled-echo[GRE], 3D GRE, susceptibility-weighted imaging, quantitative susceptibility mapping [QSM]) are more sensitive than $\mathrm{CT}$ for $\mathrm{CMH}$ detection. ${ }^{7,8}$ However, all these MR imaging CMH evaluation methods, other than QSM, ${ }^{9-13}$ will depict CMHs as bloom artifacts instead of as actual lesion size. ${ }^{14}$ They also have an appearance that varies on the basis of MR imaging field strength and scan parameters ${ }^{9,15-18}$ and require an additional phase map to distinguish blood products (diamagnetic) from calcium $^{19}$ (paramagnetic). Also, QSM distinguishes calcium from blood products better than susceptibility-weighted imaging. ${ }^{20}$ QSM estimates the intrinsic susceptibility distribution of tissue by deconvolving the blooming in the GRE phase. ${ }^{21}$

Most QSM methods, however, use a mask (mQSM) to remove the phase of low-magnitude signal to avoid singularities and streaking artifacts (mainly in the noise). The mask may either include high-susceptibility regions shown as hypointense blooming artifacts on GRE or mask out regions of brain parenchyma. Selecting a brain mask can be challenging, especially near the brain boundary, where large tissue-air or tissue-bone susceptibility differences can cause substantial signal loss on the magnitude images used to define the mask. ${ }^{21}$ This signal loss can be made even worse when hemorrhage is near the boundary. Using a mask for mQSM that is too small can include noisy phase information and lead to streaking, while a mask that is too large results in nonvisualized brain. Erosion of the brain mask can especially prevent visualization of important structures at the brain boundaries. Although numerous skull-stripping algorithms can be applied to generate an optimal mask for any individual case, there is no one automated method that will uniformly generate the optimal mask for all cases. Even optimal masks can be made suboptimal after application of extreme intensity masking by some algorithms, which can lead to nonvisualized CMHs. These mask issues can result in variable portions of brain nonvisualization, impeding the application of mQSM methods in clinical practice.

A potential solution to this problem is application of the preconditioned QSM (pQSM) method with total field inversion, ${ }^{22}$ which produces whole-head quantitative susceptibility maps without skull-stripping, reduces the error propagation associated with imprecise background field removal, and suppresses streaking artifacts in intracerebral hemorrhage on QSM images. ${ }^{22}$ pQSM calculates tissue-susceptibility values for the entire FOV (including the otherwise hypointense areas). We hypothesize that removing the need for a brain mask will enable pQSM imaging to generate robust brain susceptibility imaging, which can more reliably identify brain parenchyma and characterize CMHs relative to MQSM. To test this hypothesis, we evaluated the visualized brain parenchyma and lesions generated from a single multiecho 3D GRE MR image in a cohort of patients with TBI and controls, comparing the images of the resulting three 3D GRE magnitude images (each at a different TE), mQSM using a standard mask method, ${ }^{23}$ and pQSM.

\section{MATERIALS AND METHODS Subjects}

Under an institutional review board protocol approved by the VA Palo Alto Health Care System and Stanford University, subjects with a history of TBI and control subjects without TBI were recruited through the Santa Clara Valley Medical Center, VA Palo Alto Health Care System, and surrounding communities. All subjects were evaluated for a history of prior TBI using the Ohio State University Traumatic Brain Injury Identification Method score (Ohio TBI score), which was then used to classify subjects as having no, mild, moderate, or severe TBI. ${ }^{24}$ Subjects also underwent neuroimaging.

A total of 81 subjects were recruited, of which only 63 subjects could tolerate the entire MR imaging protocol to undergo multiecho 3D GRE for QSM imaging. Of these 63 subjects, mQSM and PQSM images could only be produced for 56 subjects $(89 \%)$ due to erroneous lines of $k$-space present in the raw data files. Subjects' ages, sex, and TBI demographic information are summarized in Table 1. There was no significant difference between non-TBI subjects and TBI subgroups (mild, moderate, or severe, as defined from the Ohio TBI score $\left.{ }^{24}\right)$ for age ( 1 -way ANOVA, $F[3,52]=0.74, P=.53)$ or $\operatorname{sex}\left(\chi^{2}=0.17\right.$, $P=.98$, Table 1$)$.

\section{Image Acquisition}

Images were obtained with a Discovery MR750 3T MR imaging scanner (GE Healthcare, Milwaukee, Wisconsin), using a 3D 
multishot multiecho EPI acquisition $(\mathrm{TR}=98 \mathrm{~ms}$; echoes $=3$; $\mathrm{TE}=14.3-19.7 / 33.1-46.99 / 51.8-74.28 \mathrm{~ms}$; matrix $=224 \times$ 224 ; resolution $=1 \times 1 \times 1 \mathrm{~mm}^{3}$ ), and a $3 \mathrm{D}$ fast-spoiled gradient-recalled acquisition $(\mathrm{TR} / \mathrm{TE} / \mathrm{TI}=9.5 / 3.8 / 900 \mathrm{~ms}$, resolution $=1 \times 1 \times 1 \mathrm{~mm}^{3}$ ) using an 8 -channel Signa head coil (GE Healthcare). Multicoil phase reconstruction was performed using the method of Bernstein et al. ${ }^{25}$ Magnitude 3D GRE images were produced automatically with the MR imaging scanner. $\mathrm{mQSM}$ images were created with the MEDI toolbox using the default mask (genmask.m). ${ }^{23}$ pQSM images were created using the method described by Liu et al. ${ }^{22,26}$

Table 2: 3D GRE, mQSM, and pQSM image review results $(n=56)$

\begin{tabular}{lcr}
\multicolumn{1}{c}{ Feature } & Count & $\%$ \\
\hline Blood products & & \\
3D GRE & 10 & 18 \\
mQSM & 4 & 7 \\
pQSM & 10 & 18 \\
Nonvisualized brain tissue on & & \\
mQSM, pQSM visible & 31 & 55 \\
mQSM and pQSM, less on pQSM & 5 & 9 \\
pQSM, mQSM visible & 0 & 0 \\
Pons probable artifacts, more visible on & & \\
pQSM & 6 & 11 \\
mQSM & 0 & 0 \\
\hline
\end{tabular}
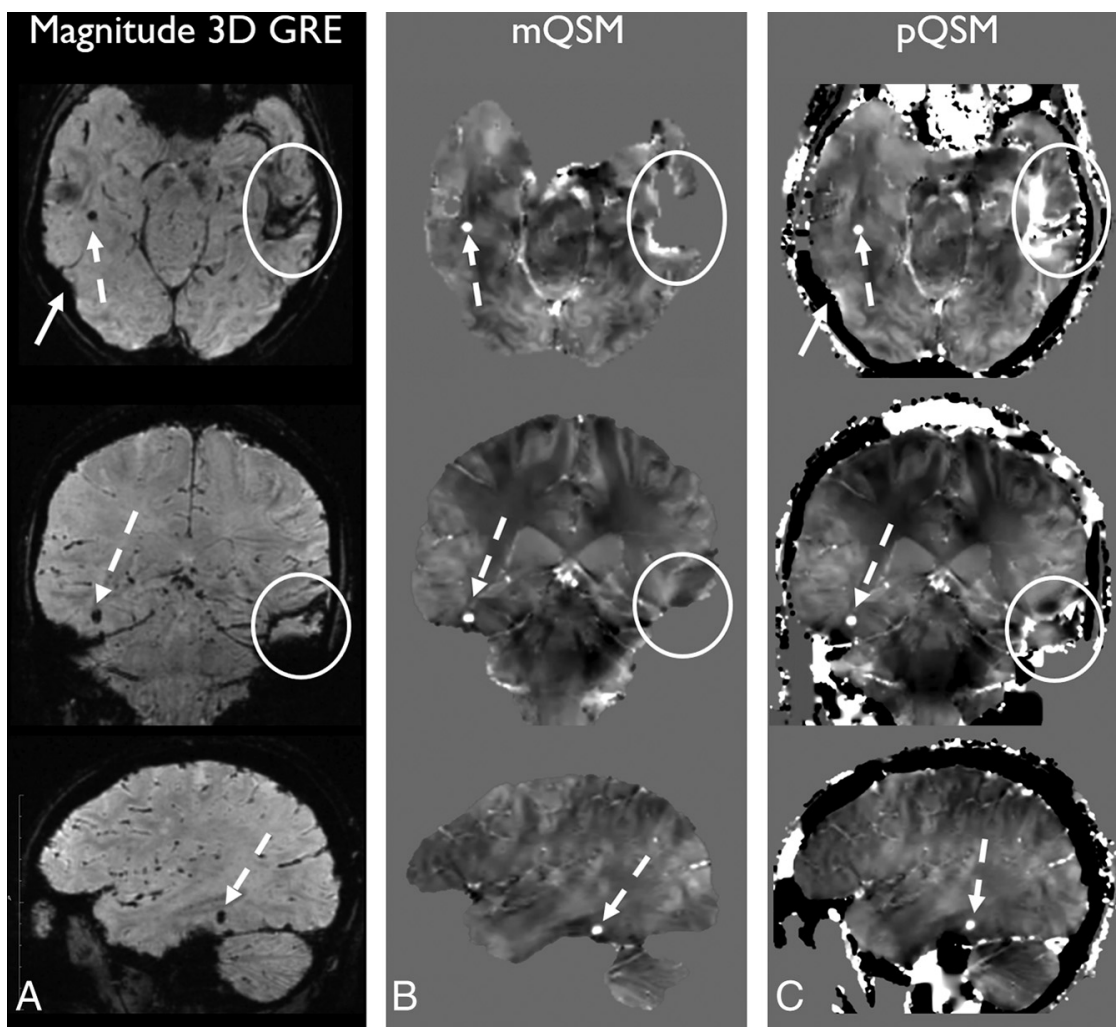

FIG 1. Paramagnetic right temporal hemorrhage is hypointense on magnitude 3D GRE (TE $=17.3$ $\mathrm{ms})(A)$ and hyperintense on $\mathrm{mQSM}(B)$ and PQSM $(C)$ images (white dashed arrows). The diamagnetic calcification in bone (white solid arrows) is mostly hypointense on 3D GRE $(A)$ and PQSM (C) and not present on mQSM $(B)$ secondary to masking. Some areas of hyperintensity within the bone may represent diamagnetic structures, such as veins or artifacts. Left temporal lobe parenchyma next to hemorrhage is eroded on the MQSM $(B)$, but preserved on magnitude and PQSM (C) images (white circles).

\section{Image Interpretation}

The images derived from the 3D multiecho GRE (the three 3D GRE magnitude volumes-each at 1 of $3 \mathrm{TE}$ values [TE $=14.3-$ 19.7/33.1-46.99/51.8-74.28 ms], the mQSM, and pQSM) and the T1-weighted fast-spoiled gradient-recalled images were examined by a neuroradiologist and a radiology resident, evaluating the presence of lesions and absent/distorted brain parenchyma, blinded to patient TBI status. Images were reviewed in 4 sessions (the $\mathrm{T} 1$ images were available for all sessions): 1) the three 3D GRE magnitude volumes, 2) the three 3D GRE volumes and mQSM, 3) the three 3D GRE volumes and pQSM images, and 4) all images. For each subject, consensus reading by the neuroradiologist and radiology resident resulting in scores of yes or no were created for the image pair attributes listed in Tables 1 and 2 .

\section{Statistics}

The Student $t$ test, ANOVA, and $\chi^{2}$ statistics with contingency tables were used to calculate statistical differences between groups on the following variables: age, sex, a history of TBI, blood on 3D GRE, and tissue missing on mQSM that was preserved on pQSM. The Fisher exact test was performed on the detection of blood on PQSM that was not seen on $\mathrm{mQSM}$ and blood on $\mathrm{mQSM}$ that was preserved on $\mathrm{pQSM}$.

\section{RESULTS}

Subjects with blood products did not show a significant difference from those without regarding age $(P=$ $.49)$, $\operatorname{sex}\left(\chi^{2}=0.04, P=.86\right.$, Table 1$)$, or TBI severity $(P=.11)$. Ten subjects demonstrated blood products on at least 1 of the three 3D GRE volumes in the occipital, temporal, parietal, or frontal lobes, as well as the basal ganglia or cerebellum (Figs 1-3). Nine of these 10 subjects had a history of TBI.

Of the 10 subjects with blood on a 3D GRE image, these cases had pQSM images depicting those lesions, but only 4 of these lesions were visible on the $\mathrm{mQSM}$ images, due to nonvisualized brain parenchyma, which was a statistically significant difference $(P<.01)$.

Thirty-one subjects (55\%) demonstrated brain parenchyma (some containing lesions) preserved on pQSM but eroded on mQSM due to masking. Five subjects $(9 \%)$ demonstrated areas of more nonvisualized brain parenchyma on $\mathrm{MQSM}$ compared with that same region on pQSM images $(P=.88$, Table 1 and Fig 3). In subjects in whom there was brain parenchyma nonvisualization on mQSM or pQSM, none demonstrated greater areas of nonvisualized brain parenchyma on PQSM relative to $\mathrm{mQSM}$ images. Overall, there was more brain parenchyma visualization on $\mathrm{pQSM}$ images compared with corre- 

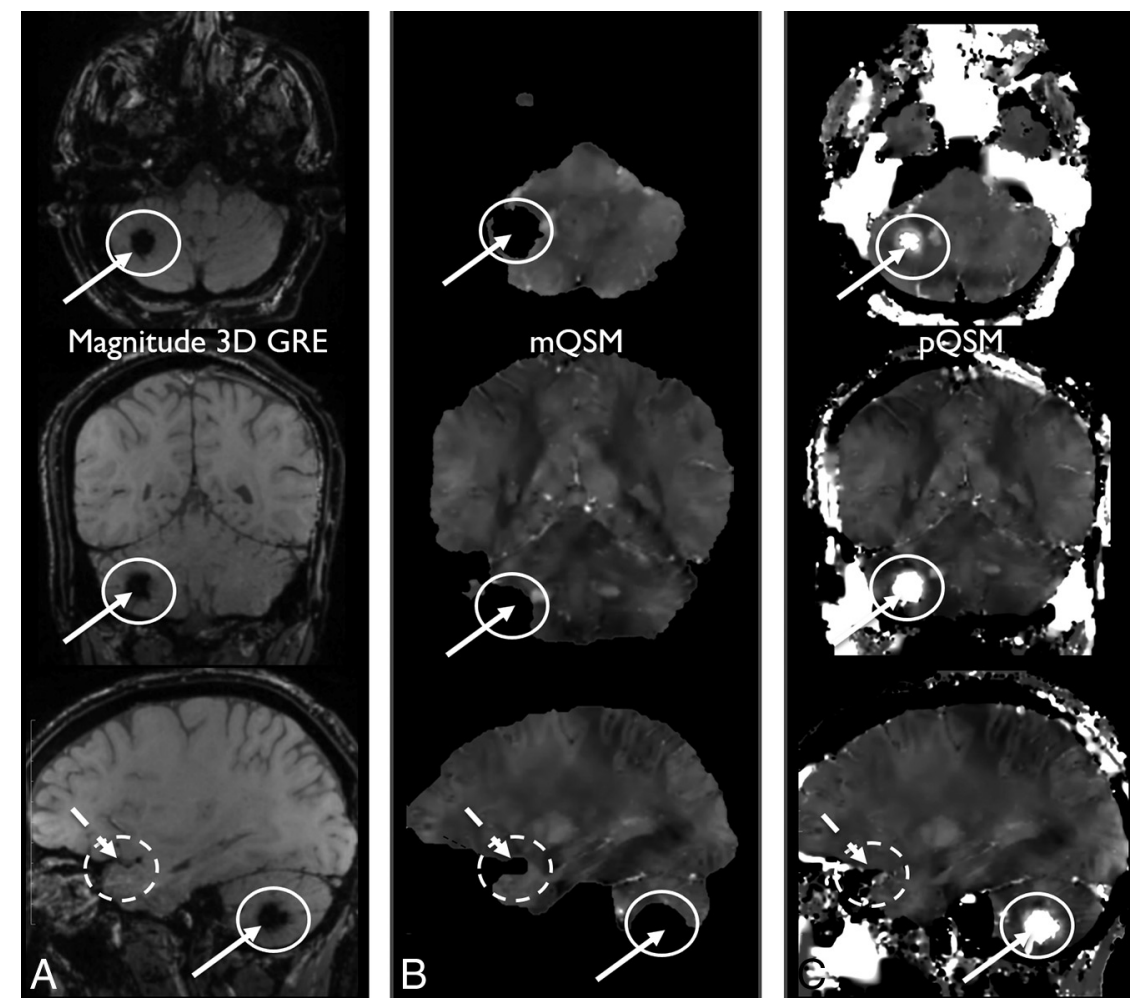

paring these images generated from a single multiecho 3D GRE scan allowed evaluation of imaging features while holding other acquisition parameters constant (eg, field strength, flip angle, sampled TEs, and so forth.). Additionally, our results demonstrate that some of these areas of brain visible on pQSM but not present on mQSM contained clinically relevant pathology, such as CMHs. This difference in brain depiction is consistent with the expectation that variations in skull-stripping performance and variations of intensity that threshold-based masking algorithms use, especially near hemorrhages (as demonstrated in Figs 2 and 3), can result in areas of nonvisualized brain on mQSM methods. This contrasts with pQSM, which uses magnitude information to differentiate weak from strong sources of susceptibility over the entire head for reconstruction. Instead of splitting the QSM problem into background field removal and local field fitting (in which errors are propagated from the first into the second), preconditioned QSM solves them together. Demonstration of better preservation of brain parenchyma in pQSM compared with mQSM in subjects was independent of age, sex, and the presence of hemorrhage or history of prior trauma; this finding suggests generalizability across patients. The preservation of anatomic landmarks, such as skull margins, on pQSM images also has the potential to aid in clinical interpretation. The feature of preserving skull and scalp in pQSM may also allow better depiction of calvarial and skin masses if challenges regarding scalp SNR and implementation of a robust water/fat separation algorithm can be managed.

sponding $\mathrm{mQSM}$ images. The differences in parenchymal depiction on pQSM compared with mQSM were statistically significant $(P<$ $.01)$. There was not a statistically significant difference in age $(P=$ $.76)$, sex $\left(\chi^{2}=0.04, P=.84\right)$, TBI severity $\left(\chi^{2}=2.20, P=.5\right.$, Table 1 ), or the presence of blood products on 3D GRE $\left(\chi^{2}=2.99, P=.08\right.$, Table 1) for cases demonstrating nonvisualized brain parenchyma on mQSM relative to cases that did not. Six subjects (11\%) demonstrated $\mathrm{MQSM}$ and $\mathrm{PQSM}$ pons hypointensity with no corresponding 3D GRE finding suggestive of artifacts, all larger on pQSM than on corresponding mQSM images (Fig 4).

\section{DISCUSSION}

This report shows that pQSM can depict more brain parenchyma than masked-based QSM using the same acquisition data. Com-

Like other neuroimaging studies evaluating cerebral microhemorrhage presence, this study used an accepted GRE imaging sequence as the reference standard for CMH presence. , $^{8,27,28}$ Here, the 3 echoes of the 3D GRE magnitude images were used as ground truth for the presence of CMHs, given that direct pathologic correlation was not possible (as is the case in many clinical imaging studies). Across the 3 TE volumes, more subtle susceptibility changes are likely to be visible at higher TEs, but often with corresponding increased artifacts. The three 3D GRE magnitude whole-brain volumes were each interpreted as they would be clinically for the presence of a lesion across the 3 TEs. However, in routine clinical practice, only 1 volume would likely be reviewed, leaving the possibility that smaller or subtler CMHs may have 

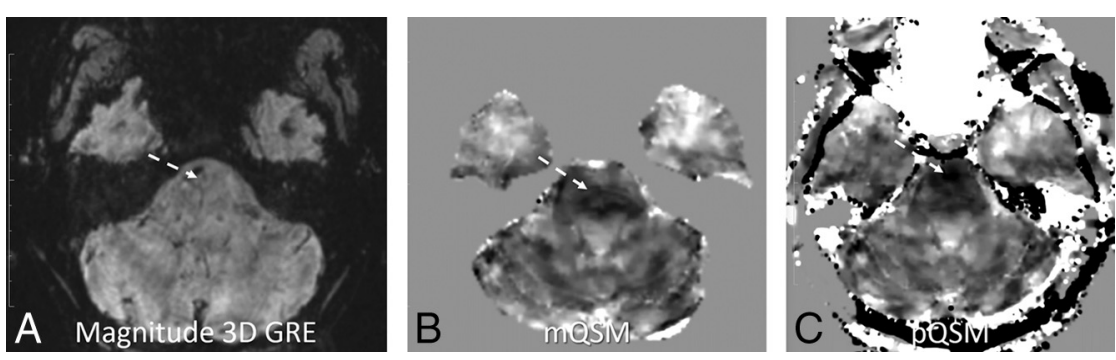

despite having 34 subjects (61\%) with a history of TBI. Future studies would benefit from larger subject cohorts, with more subjects demonstrating susceptibility-based pathologies, such as CMHs. An additional limitation is that categorization of brain loss in the current study was performed by only 2 readers providing a consensus read, and subsequent

FIG 4. PQSM $(C)$ demonstrates worse nonspecific diffuse low signal in the pons compared with mQSM $(B)$, with no corresponding abnormality on the magnitude 3D GRE (TE $=41.8 \mathrm{~ms}$ ) image $(A)$ (white dashed arrows). Note that areas of hypointensity are in different positions within the pons.

studies would benefit from using an automated or multireader evaluation. The 6 cases $(11 \%)$ in which pQSM demonstrated greater pontine tissue contrast relative to $\mathrm{mQSM}$, with no definite cor-

been visible at a longer TE, which is not the case for QSM-based images. Also, reviewing those magnitude images without phase maps would leave room for doubt as to whether the lesions were blood products or calcification, as is often the case in current clinical practice. Because the PQSM images did not show any lesions that did not appear on at least 1 of the 3D GRE images, no false-positives were encountered.

While our work found PQSM to always depict more brain parenchyma than $\mathrm{mQSM}$, there was still omission of some brain areas on PQSM relative to at least 1 of the 3 TE 3D GRE images. These areas of pQSM failure may be related to the magnitude image of the first echo (14.3-19.7 ms in this study) not being sufficiently short, impacting the ability of PQSM to differentiate strong and weak sources. At this level of first TE, the cortex might already experience R2* decay due to field inhomogeneity at air/ tissue interfaces. QSM may only reduce blooming artifacts to the amount in the first echo. Future work should study the ability of a shorter first TE to remedy this problem. The instances in which pQSM demonstrates brain parenchyma more than mQSM, but with some distortion or some nonvisualized brain tissue, do raise the concern about whether it is better to not demonstrate tissue at all rather than show it with distortion. Future work incorporating lower TE information should consider this question as well.

Overall, the osseous structures on QSM appear hypointense, compatible with paramagnetic calcification. However, some hyperintense areas in bone were noted (Figs 1-4). These findings may be related to intraosseous venous structures or artifacts (because, in the case of skull bone, there is little local phase signal to aid in identifying its susceptibility value). Although the current implementation of pQSM is not optimized for bone, improvement could be made by acquiring the first echo at an earlier TE, similar to acquisitions such as ultrashort TE, to more reliably characterize bone properties.

This study did not evaluate the quantitative aspects of pQSM. Although QSM is a quantitative method, current clinically used susceptibility-based neuroimaging methods are interpreted solely on a qualitative basis, assessing hypointense foci, with the occasional addition of phase maps to distinguish blood from calcification. Future work could assess the additional clinical value of $\mathrm{mQSM} / \mathrm{pQSM}$ quantitative lesion analysis.

Limitations of this study include the small sample size of patients with hemorrhage. While this work did not clearly demonstrate instances of CMHs visible on PQSM or mQSM that were not seen on 3D GRE images, only 10 (18\%) subjects had CMHs, responding anatomic or pathologic finding on 3D GRE images (Fig 4), also represent an important area for future evaluation. Further investigation of pQSM specificity for CMH evaluation would also advance defining the clinical role that pQSM can play in managing patients in whom identifying $\mathrm{CMHs}$ is clinically relevant.

\section{CONCLUSIONS}

pQSM can improve the evaluation of $\mathrm{CMH}$-associated pathologies, such as TBI.

Disclosures: Stephanie Kolakowsky-Hayner-UNRELATED: Consultancy: Gerson Lehrman Group, Comments: various consulting calls related to brain injury rehabilitation; Employment: Ichan School of Medicine at Mount Sinai, Comments: current employer; Grants/Grants Pending: National Institute on Disability, Independent Living, and Rehabilitation Research, Comments: TBI Model Systems Grant, Spinal Cord Injury Model Systems Grant, Spinal Cord Injury Model Systems Collaborative Grant*; Payment for Lectures Including Service on Speakers Bureaus: Brain Injury Association of Virginia, Comments: stipend for keynote; Royalties: Lash and Associates, Comments: Tip Card Royalties; Travel/Accommodations/Meeting Expenses Unrelated to Activities Listed: Paralyzed Veterans of America, Comments: conference scholarship. Maheen M. Adamson-RELATED: Grant: VA Office of Public Health (War Related Illness and Injury Study Center)*; Support for Travel to Meetings for the Study or Other Purposes: VA Office of Public Health (War Related Illness and Injury Study Center)*. Pascal Spincemallie-RELATED: Grant: National Institutes of Health, Comments: R01CA181566, includes salary support*; Support for Travel to Meetings for the Study or Other Purposes: National Institutes of Health, Comments: R01CA181566*; UNRELATED: Patents (Planned, Pending or Issued): US 8781197*. Yi Wang-RELATED: Grant: National Institutes of Health, Comments: R01CA178007, R01NS090464, R01NS095562*; UNRELATED: Patents (Planned, Pending or Issued): Cornell University, Comments: I am one of the inventors on QSM technology*; Stock/Stock Options: Medimagemetric, Comments: Medimagemetric is working with Cornell on commercializing QSM technology.* Magdy SelimUNRELATED: Grants/Grants Pending: National Institute of Neurological Disorders and Stroke, American Heart Association*; Royalties: Oxford University Press, Cambridge University Press. *Money paid to the institution.

\section{REFERENCES}

1. Xi G, Keep RF, Hoff JT. Mechanisms of brain injury after intracerebral haemorrhage. Lancet Neurol 2006;5:53-63 CrossRef Medline

2. Greenberg SM, Vernooij MW, Cordonnier C, et al; Microbleed Study Group. Cerebral microbleeds: a guide to detection and interpretation. Lancet Neurol 2009;8:165-74 CrossRef Medline

3. Akoudad S, Portegies ML, Koudstaal PJ, et al. Cerebral microbleeds are associated with an increased risk of stroke: the Rotterdam Study. Circulation 2015;132:509-16 CrossRef Medline

4. Charidimou A, Kakar P, Fox Z, et al. Cerebral microbleeds and recurrent stroke risk: systematic review and meta-analysis of prospective ischemic stroke and transient ischemic attack cohorts. Stroke 2013;44:995-1001 CrossRef Medline

5. Mehndiratta P, Manjila S, Ostergard T, et al. Cerebral amyloid angi- 
opathy-associated intracerebral hemorrhage: pathology and management. Neurosurg Focus 2012;32:E7 CrossRef Medline

6. Jouvent E, Puy L, Chabriat H. Cerebral microhemorrhages: significance, associations, diagnosis, and treatment. Curr Treat Options Neurol 2016;18:35 CrossRef Medline

7. Mittl RL, Grossman RI, Hiehle JF, et al. Prevalence of MR evidence of diffuse axonal injury in patients with mild head injury and normal head CT findings. AJNR Am J Neuroradiol 1994;15:1583-89 Medline

8. Kidwell CS, Chalela JA, Saver JL, et al. Comparison of MRI and CT for detection of acute intracerebral hemorrhage. JAMA 2004;292: 1823-30 CrossRef Medline

9. de Rochefort L, Liu T, Kressler B, et al. Quantitative susceptibility map reconstruction from MR phase data using Bayesian regularization: validation and application to brain imaging. Magn Reson Med 2010;63:194-206 CrossRef Medline

10. Haacke EM, Tang J, Neelavalli J, et al. Susceptibility mapping as a means to visualize veins and quantify oxygen saturation. J Magn Reson Imaging 2010;32:663-76 CrossRef Medline

11. Liu T, Xu W, Spincemaille P, et al. Accuracy of the morphology enabled dipole inversion (MEDI) algorithm for quantitative susceptibility mapping in MRI. IEEE Trans Med Imaging 2012;31: 816-24 CrossRef Medline

12. Schweser F, Sommer K, Deistung A, et al. Quantitative susceptibility mapping for investigating subtle susceptibility variations in the human brain. Neuroimage 2012;62:2083-100 CrossRef Medline

13. Neelavalli J, Cheng YC, Jiang J, et al. Removing background phase variations in susceptibility-weighted imaging using a fast, forwardfield calculation. J Magn Reson Imaging 2009;29:937-48 CrossRef Medline

14. Liu T, Surapaneni K, Lou M, et al. Cerebral microbleeds: burden assessment by using quantitative susceptibility mapping. Radiology 2012;262:269-78 CrossRef Medline

15. Nandigam RN, Viswanathan A, Delgado P, et al. MR imaging detection of cerebral microbleeds: effect of susceptibility-weighted imaging, section thickness, and field strength. AJNR Am J Neuroradiol 2009;30:338-43 Medline

16. Soman S, Bregni JA, Bilgic B, et al. Susceptibility-based neuroimaging: standard methods, clinical applications, and future directions. Curr Radiol Rep 2017;5 pii: 11 CrossRef Medline

17. Schweser F, Deistung A, Lehr BW, et al. Differentiation between dia- magnetic and paramagnetic cerebral lesions based on magnetic susceptibility mapping. Med Phys 2010;37:5165-78 CrossRef Medline

18. Li J, Chang S, Liu T, et al. Reducing the object orientation dependence of susceptibility effects in gradient echo MRI through quantitative susceptibility mapping. Magn Reson Med 2012;68:1563-69 CrossRef Medline

19. Chen W, Zhu W, Kovanlikaya I, et al. Intracranial calcifications and hemorrhages: characterization with quantitative susceptibility mapping. Radiology 2014;270:496-505 CrossRef Medline

20. Ciraci S, Gumus K, Doganay S, et al. Diagnosis of intracranial calcification and hemorrhage in pediatric patients: comparison of quantitative susceptibility mapping and phase images of susceptibility-weighted imaging. Diagn Interv Imaging 2017;98:707-14 CrossRef Medline

21. Wang Y, Liu T. Quantitative susceptibility mapping (QSM): decoding MRI data for a tissue magnetic biomarker. Magn Reson Med 2015;73:82-101 CrossRef Medline

22. Liu Z, Kee Y, Zhou D, et al. Preconditioned total field inversion (TFI) method for quantitative susceptibility mapping. Magn Reson Med 2017;78:303-15 CrossRef Medline

23. Liu J, Liu T, de Rochefort L, et al. Morphology enabled dipole inversion for quantitative susceptibility mapping using structural consistency between the magnitude image and the susceptibility map. Neuroimage 2012;59:2560-68 CrossRef Medline

24. Rapp PE, Rosenberg BM, Keyser DO, et al. Patient characterization protocols for psychophysiological studies of traumatic brain injury and post-TBI psychiatric disorders. Front Neurol 2013;4:91 CrossRef Medline

25. Bernstein MA, Grgic M, Brosnan TJ, et al. Reconstructions of phase contrast, phased array multicoil data. Magn Reson Med 1994;32: 330-34 CrossRef Medline

26. Liu Z, Wen Y, Spincemaille P, et al. Optimization of preconditioned total field inversion for whole head QSM and cardiac QSM. In: Proceedings of the International Society for Magnetic Resonance in Medicine Annual Meeting and Exhibition, Honolulu, Hawaii. April 22-27, 2017

27. Vernooij MW, Ikram MA, Wielopolski PA, et al. Cerebral microbleeds: accelerated $3 \mathrm{D}$ T2 ${ }^{*}$-weighted GRE MR imaging versus conventional 2D T2* -weighted GRE MR imaging for detection. $R a$ diology 2008;248:272-77 CrossRef Medline

28. Soman S, Holdsworth SJ, Barnes PD, et al. Improved T2* imaging without increase in scan time: SWI processing of $2 \mathrm{D}$ gradient echo. AJNR Am J Neuroradiol 2013;34:2092-97 CrossRef Medline 